\title{
Sonography of Ruptured Appendicitis
}

\author{
Caroline E. Blane, ${ }^{1}$ Susan J. White, ${ }^{1}$ John R. Wesley, ${ }^{2}$ and Arnold G. Coran ${ }^{2}$ \\ ${ }^{1}$ Department of Radiology, Section of Pediatric Radiology, and ${ }^{2}$ Department of Surgery, Section of Pediatric Surgery, \\ C.S. Mott Children's Hospital, The University of Michigan, Ann Arbor, Michigan, USA
}

\begin{abstract}
Three girls and 4 boys with the final diagnosis of ruptured appendicitis underwent 19 sonographic studies, 5 before any therapy was instituted and 14 follow-up studies. Four abscesses identified before treatment and 2 seen postoperatively were ovoid, irregularly marginated, and contained lowamplitude echoes. Gastrointestinal ileus with gasand fluid-filled loops of bowel made the studies technically difficult. Follow-up studies also showed fluid collections distant from the original abscesses. The complete resolution of the abscess was followed sonographically in 1 patient treated with antibiotics. Ultrasound can be useful in confirming or suggesting the diagnosis of ruptured appendicitis in the child with abdominal pain, but careful attention to detail is necessary in these technically difficult studies.
\end{abstract}

Key words: Ultrasound - Appendicitis - Abscess, diagnosis.

The most common surgical problem encountered in childhood is appendicitis [1]. Appendicitis was reported as a distinct entity in 1759 but it was not until 1887 that the first laparotomy was performed for treatment and removal of the diseased appendix [1]. Despite the recognized frequency of appendicitis, it is responsible for the greatest number of errors in surgical diagnosis [1]. This is due in part to the initial presentation with abdominal pain that can vary with respect to severity and location. Abdominal pain is a frequent com-

Address reprint requests to: C. Blane, M.D., Department of Radiology, C.S. Mott Children's Hospital, University Hospitals, Ann Arbor, MI 48109-0010, USA plaint in childhood, and the differential diagnosis embraces a large group of conditions, including viral gastroenteritis. Ruptured appendicitis usually presents with abdominal pain but the constellation of findings may confuse the clinician and the diagnosis may not be suspected. Children with a history of generalized abdominal pain often undergo an ultrasound examination as part of the initial evaluation. Thus, ultrasound can be the initial test to suggest the diagnosis of ruptured appendicitis. The variety of sonographic appearances of ruptured appendix is reviewed.

\section{Materials and Methods}

Between January 1983 and May 1985, 7 patients were seen at our hospital with a final diagnosis of ruptured appendicitis, all of whom had undergone abdominal and pelvic ultrasound studies. All ultrasound examinations included studies with a real-time mechanical sector scanner with a $5-\mathrm{MHz}$ transducer, medium range focus. Three patients also had static $B$ scans performed with a Phillips 3600 with a $3.5-\mathrm{MHz}$ transducer. All sonograms were analyzed retrospectively by 2 of the authors. The clinical course, operative procedures, and pathologic findings were obtained from chart review.

Three girls and 4 boys were examined; they ranged in age from 5 to 16 years. All had a history of abdominal pain, the duration of which varied from 2 to 12 days with a mean of 5 days at the time of presentation to the surgical staff. Once these children were seen by the surgeons, treatment was instituted within 24 hours. One child had been seen twice over a 5-day period with abdominal pain, diarrhea, and vomiting before the diagnosis was suggested and the child referred to the surgical staff. Another child with a dense hemiparesis, congenital heart disease, and right-sided abdominal pain had 2 ultrasound studies 4 and 2 days before needle aspiration of an abdominal mass confirmed the diagnosis. Two children had been presumed to have viral gastroenteritis for 48 hours before the correct diagnosis was entertained. Five children had a laparotomy during the acute stage of the illness (1 at another institution). One child was treated with antibiotics and readmitted at a later date for appendectomy. 

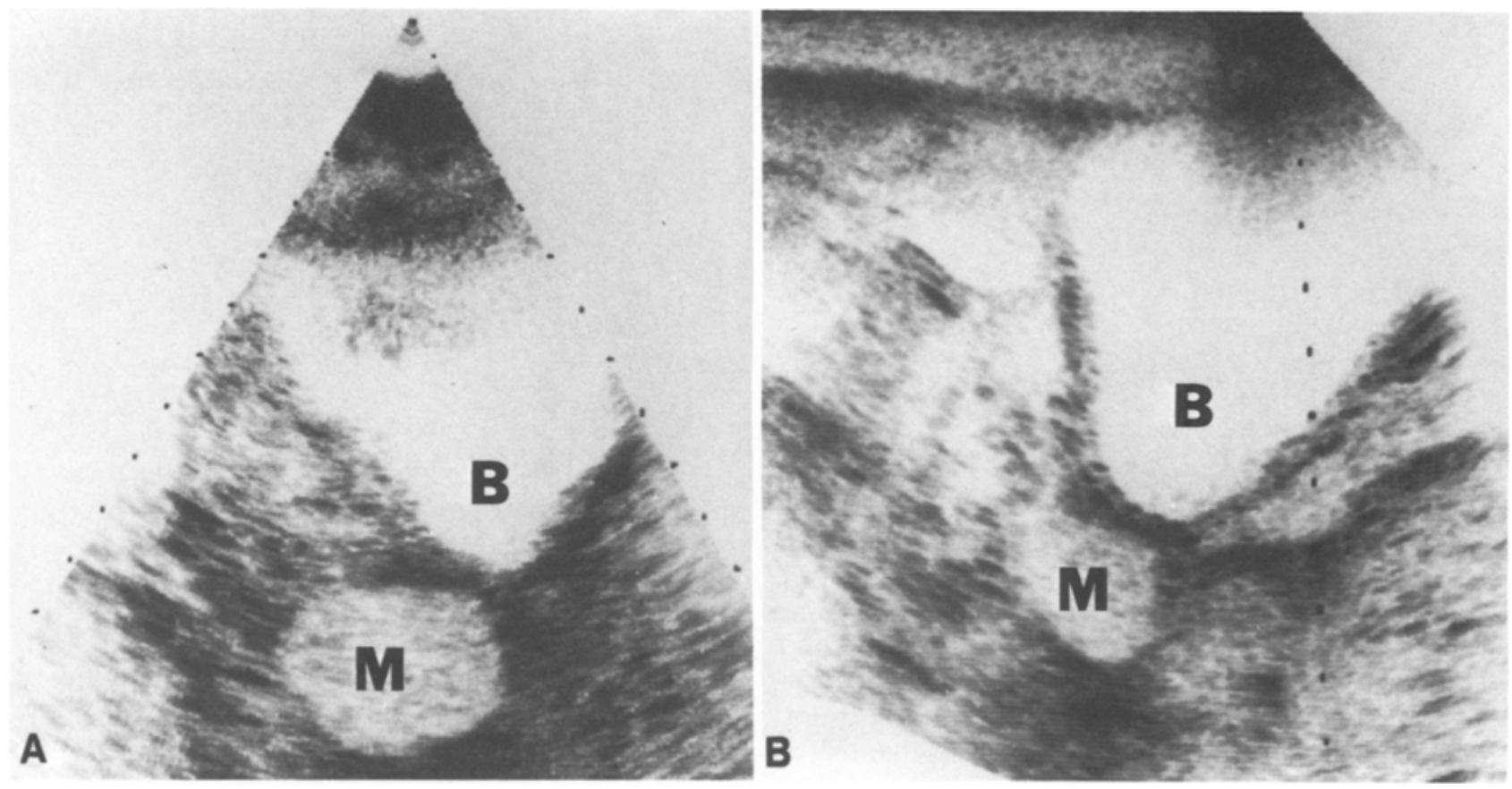

Fig. 1. Transverse (A) and longitudinal (B) sonograms of the pelvis of a 16-year-old show a complex mass ( $M$ ) posterior to the bladder $(B)$. Enhanced transmission of sound is demonstrated behind the mass (abscess) lesion, which contains low-amplitude echoes.

\section{Results}

Four patients whose sonograms were taken prior to treatment had mass lesions demonstrated. Three abscesses were situated deep in the pelvis behind the bladder and the fourth was anterior and to the right of the bladder just beneath the skin. These masses were all ovoid, $4-5 \mathrm{~cm}$ in average dimension, with irregular margins and low-amplitude internal echoes (Fig. 1). Enhanced through-transmission of sound was demonstrated in only 2 cases, with equivocal demonstration in the other 2 examinations because of adjacent air-filled bowel. All the studies were technically complicated by ileus associated with excessive intestinal gas and fluidfilled bowel loops. One abscess was overlooked on the first ultrasound examination because it was confused with the rectum despite administration of a water enema.

One child treated with antibiotics primarily was followed with serial sonograms at 5, 11, and 25 days after treatment was started. The abscess slowly decreased in size over that time and could not be identified on the final examination (Fig. 2). At that time, she was readmitted for elective appendectomy.

Four of the 6 patients who had early surgical intervention had postoperative sonograms per- formed for persistent fever. Ileus with fluid- and gas-filled loops of bowel made all of the studies technically difficult. Two of the patients had left pleural effusions. Three of the patients had localized fluid collections in various intraabdominal locations: 2 in Morrison's pouch; 3 in the subdiaphragmatic space, 2 on the left and 1 on the right; 1 in the subhepatic space; 1 at the tip of the spleen; and 1 in the pelvis (Fig. 3). Three patients had focal mass lesions that were presumed to be abscesses in the pelvis behind the bladder. These postoperative abscesses also had irregular margins, low-amplitude internal echoes, an ovoid shape, and enhanced through-transmission of sound. Both water enemas and the administration of water orally were necessary in many of these studies to define the rectum, duodenum, and stomach.

\section{Discussion}

Abscesses associated with ruptured appendicitis can be identified sonographically. However, the intestinal gas- and fluid-filled loops of bowel often associated with a generalized ileus make the task challenging. Retrovesical abscesses can be particularly difficult to define because they are confused with the rectosigmoid colon. We found the abscesses to be ovoid with irregular margins. The back 

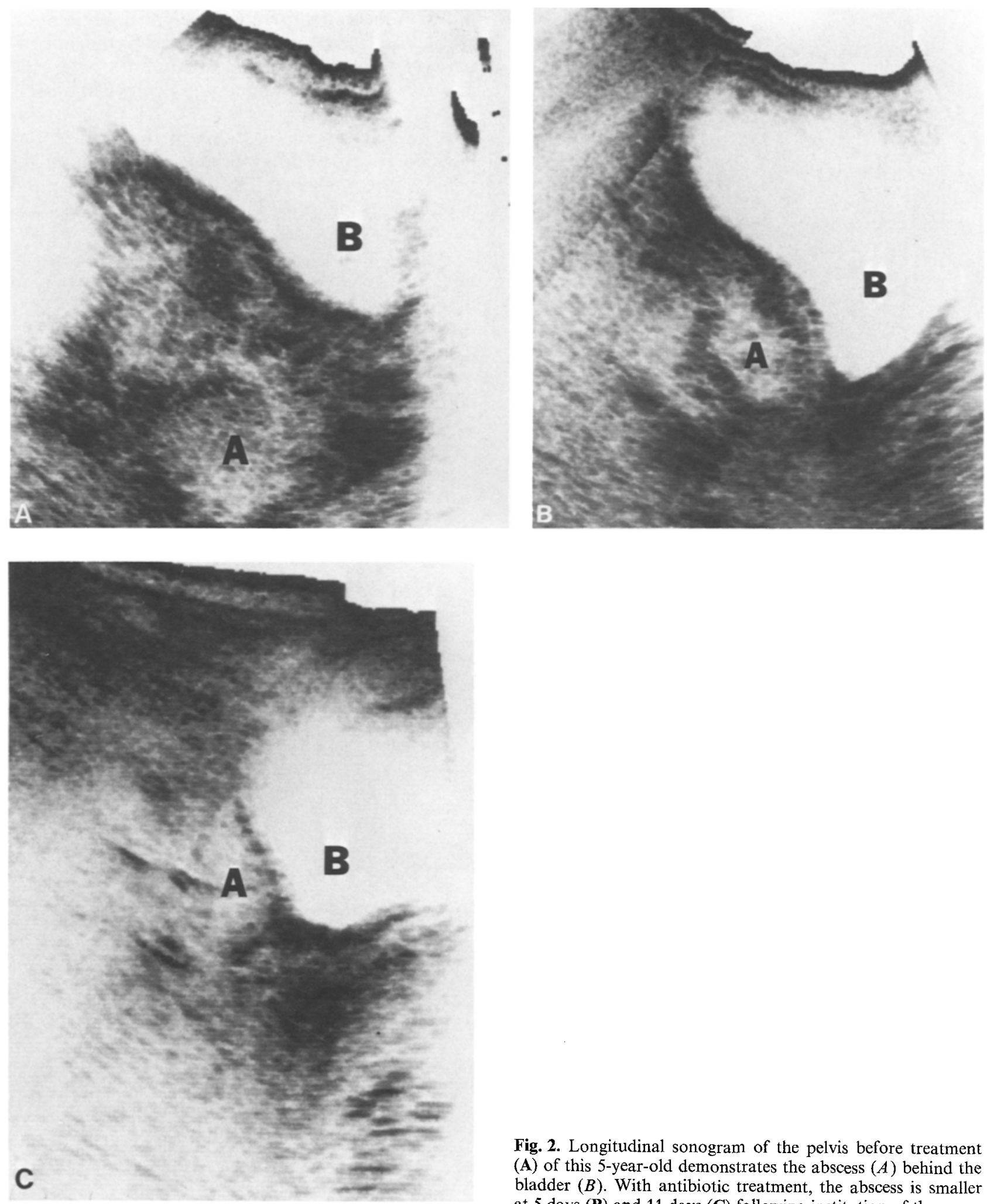

Fig. 2. Longitudinal sonogram of the pelvis before treatment (A) of this 5-year-old demonstrates the abscess $(A)$ behind the bladder $(B)$. With antibiotic treatment, the abscess is smaller at 5 days $(\mathbf{B})$ and 11 days (C) following institution of therapy. 


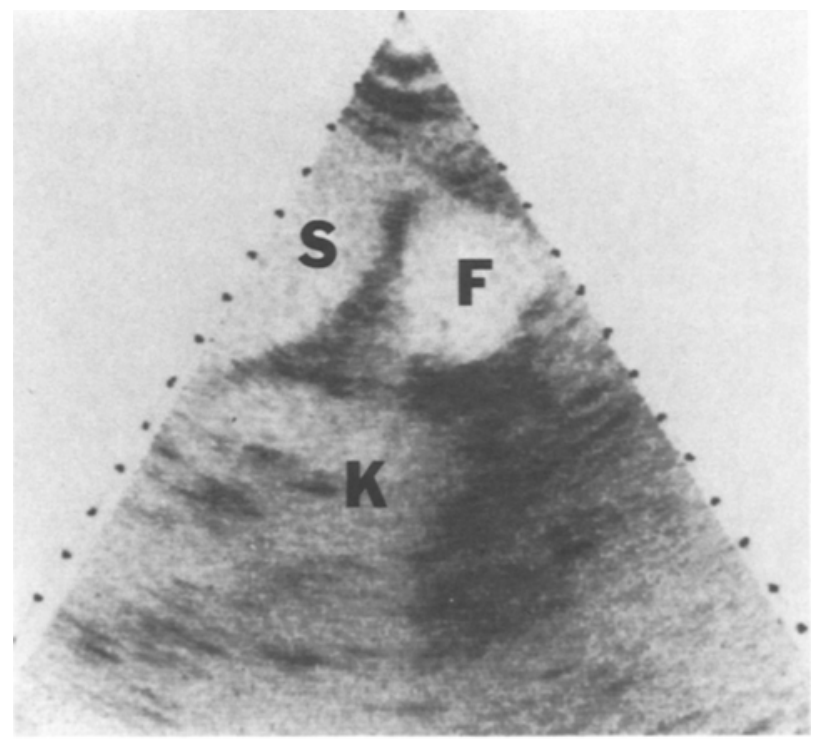

Fig. 3. A longitudinal sonogram of the left flank of this 7-yearold boy following surgery for perforated appendix shows a locular fluid collection $(F)$ anterior to the left kidney $(K)$ at the tip of the spleen $(S)$.

walls were not sharply defined. They contained low-amplitude internal echoes (Figs. 1 and 2). This is in contrast to previous published reports of abscesses with appendicitis, which have been reported to be hypoechoic, echo-free, or "cystic" mass lesions [2-4]. However, in a general review of abdominal abscesses, Filly describes diffuse low-amplitude internal echoes as one sonographic feature of abdominal abscesses with a wide variety of sonographic appearances [3]. We did not see "cystic" or echo-free periappendiceal abscesses in our patients. Sonography was used to assess abscess reso- lution in the patient treated solely with antibiotics. Antibiotic therapy could be tailored to the individual patient in this case and discontinued when the abscess was no longer sonographically demonstrable.

The diversity of locations of fluid collections postoperatively in these patients with ruptured appendix is emphasized. Not only were localized mass lesions identified but locular fluid collections in other distant areas were also seen.

Ultrasound is often the initial imaging procedure in a child presenting with abdominal pain of unknown cause. Since early diagnosis of ruptured appendicitis is the most important single factor in reducing the morbidity and mortality in this condition, it is important to appreciate the sonographic presentation. Ultrasound can be useful in confirming the diagnosis and following the abscesses to resolution, but careful attention to detail is necessary in these technically difficult studies.

\section{References}

1. Martin L: Appendicitis. In Mustard WT, Ravitch MM, Snyder WH Jr. et al. (eds): Pediatric Surgery, vol 2, 2nd ed. Chicago: Yearbook Medical Publishers, 1972, pp 948-959

2. Doust BD, Quiroz F, Stewart JM: Ultrasonic distinction of abscesses from other intra-abdominal fluid collections. $R a$ diology $125: 213-218,1977$

3. Filly RA: Detection of abdominal abscesses: a combined approach employing ultrasonography, computed tomography and gallium-67 scanning. $J$ Can Assoc Radiol $30: 202-210,1979$

4. Parulekar SG: Ultrasonographic findings in diseases of the appendix. $J$ Ultrasound Med 2:59-64, 1983

Received: September 11, 1985; accepted: November 4, 1985 